\title{
Simultaneous surgery in patients with both cardiac and noncardiac diseases
}

This article was published in the following Dove Press journal:

Patient Preference and Adherence

18 July 2016

Number of times this article has been viewed

\author{
Yang Yang' \\ Feng Xiao' \\ Jin Wang' \\ Bo Song' \\ $\mathrm{Xi}-\mathrm{Hui} \mathrm{Li}^{\prime}$ \\ Jian $\mathrm{Li}^{2}$ \\ Zhi-Song $\mathrm{He}^{3}$ \\ Huan Zhang ${ }^{4}$ \\ Ling Yin ${ }^{5}$ \\ 'Department of Cardiac Surgery, \\ ${ }^{2}$ Department of Thoracic Surgery, \\ ${ }^{3}$ Department of Urology Surgery, \\ ${ }^{4}$ Department of General Surgery, \\ ${ }^{5}$ Department of Obstetrics and \\ Gynecology, Peking University \\ First Hospital, Beijing, People's \\ Republic of China
}

Correspondence: Yang Yang

Department of Cardiac Surgery, Peking University First Hospital, No 8 Xishiku Street, Beijing 100034, People's Republic of China Tel +8610 83572744

Email yangyang_12456@I26.com
Background: To investigate the possibility and feasibility of simultaneous cardiac and noncardiac surgery.

Methods: From August 2000 to March 2015, 64 patients suffering from cardiac and noncardiac diseases have been treated by simultaneous surgeries.

Results: Two patients died after operations in hospital; thus, the hospital mortality rate was $3.1 \%$. One patient with coronary heart disease, acute myocardial infarction, and a recurrence of bladder cancer accepted emergency simultaneous coronary artery bypass grafting (CABG), bladder cystectomy, and ureterostomy. He died of acute cerebral infarction complicated with multiple organ failure on the 153rd day after operation. The other patient with chronic constrictive pericarditis and right lung cancer underwent pericardial stripping and right lung lower lobectomy, which resulted in multiple organ failure, and the patient died on the tenth day postoperatively. The remaining 62 patients recovered and were discharged. The total operative morbidity was $17.2 \%$ : postoperative hemorrhage (n, \% $[1,1.6 \%]$ ), pulmonary infection and hypoxemia (2, $3.1 \%)$, hemorrhage of upper digestive tract $(1,1.6 \%)$, incisional infection $(3,4.7 \%)$, subphrenic abscess $(1,1.6 \%)$, and postoperative acute renal failure and hemofiltration $(3,4.7 \%)$. Of the 62 patients discharged, 61 patients were followed up. Eleven patients died with 10 months to 10 years during the follow-up. The mean survival time is $116.2 \pm 12.4$ months. The cumulative survival rate is $50.8 \%$.

Conclusion: Simultaneous surgeries in patients suffering from both cardiac and noncardiac benign or malignant diseases are safe and possible with satisfactory short-term and long-term survival.

Keywords: cardiac surgery, noncardiac surgery, coronary artery bypass grafting (CABG), tumor resection, simultaneous surgery

\section{Introduction}

It is very difficult to decide how to treat cardiac patients who need a noncardiac surgical operation at the same time, especially those with an early malignant tumor and severe coronary artery diseases (CADs), as the previous operations are often contraindications for each other. Fortunately, the remarkable improvement of surgical techniques makes it possible to conduct combined cardiac and noncardiac operations. From August 2000 to April 2015, the Department of Cardiac Surgery, Department of Thoracic Surgery, Department of Urology Surgery, Department of General Surgery, and Department of Gynecology of Peking University First Hospital performed simultaneous surgery of cardiac and noncardiac disease patients and observed the short-term and long-term effects of operations. This is the largest domestic single-center report in the People's Republic of China nowadays. 


\section{Methods}

\section{Patients}

The Human Ethics Committee of Peking University First Hospital approved this research. All patients were given a verbal and written explanation of the study, and no personal information was recorded during the research. All patients provided written informed consent. This study was conducted in accordance with the Declaration of Helsinki. There were 64 patients in our study, 49 males $(76.6 \%)$ and 15 females $(23.4 \%)$, aged from 41 years to 84 years (mean age $65.16 \pm 9.34$ years).

Cardiac diseases included 53 cases of CAD (nine cases of single-vessel disease and 44 cases of multivessel disease, including one case combined with left ventricular aneurysm and thrombus and one case combined with congenital heart disease ventricular defect), eight cases of heart valve disease ( six cases of rheumatic heart disease and two cases of degenerative valvular disease), one case of chronic constrictive pericarditis, and two cases of cardiac tumors.

Noncardiac diseases included the following chest diseases and abdominal diseases:

Chest diseases: Twenty-one cases of pulmonary disease (18 cases of lung cancer, one case of pulmonary hamartoma, one case of pulmonary carcinoid, and one case of pulmonary inflammatory granuloma), two cases of esophageal cancer, two cases of thymoma, two cases of thymic cyst, one case of mediastinal adenophyma, two cases of breast cancer, and one case of chest wall cavernous hemangioma.

Abdominal diseases: 14 cases of gastrointestinal diseases (three cases of gastric cancer, three cases of colon cancer, three cases of rectal cancer, one case of liver cancer, three cases of cholecystitis including one case of chronic appendicitis, and one case of biliary calculi), 14 cases of urinary cancer (nine cases of renal cancer, including three cases of inferior vena cava tumor thrombus, one case of renal pelvis cancer, and one case of adrenal cancer; one case of ureteral cancer; and two cases of bladder cancer), and five cases of gynecological diseases (four cases of endometrial cancer and one case of ovarian cyst) (Table 1).

\section{Simultaneous operation types}

The heart and lung operations included coronary artery bypass grafting (CABG) combined with left upper lobectomy in three cases; left upper lobe wedge resection in two cases; left lower lobectomy in one case; left lower lobe partial resection in one case; left pneumonectomy and left atrial tumor thrombus removal in one case; right upper lobectomy in four cases; right upper lobe sleeve resection in one case; right middle lobectomy in one case; right lower lobectomy in two cases; right lower lobe wedge resection in one case; bilateral lung metastasis cancer resection in one case; and right lung lymph node biopsy in one case; aortic and mitral bioprosthetic valve replacement and left upper lobectomy in one case; and left atrial myxoma resection and right lower lobe wedge resection in one case.

The heart and mediastinum operations included aortic bioprosthetic valve replacement and thymic cyst resection in one case; mitral bioprosthetic valve replacement and thymectomy in one case; $\mathrm{CABG}$ and thymectomy in two cases; and $\mathrm{CABG}$ and mediastinal lymph node dissection in one case.

The heart and breast cancer operations included CABG combined with ventricular septal defect repair and left modified radical mastectomy in one case and mitral valve replacement and left modified radical mastectomy in one case.

The heart and chest wall operation included aortic bioprosthetic valve replacement and left chest wall cavernous hemangioma resection in one case.

The heart and gastrointestinal tract operations included CABG and cervical anastomosis of esophagus and stomach in two cases; $\mathrm{CABG}$ and gastric cardia cancer resection in one

Table I Clinical data of combined cardiac and noncardiac disease

\begin{tabular}{lllll}
\hline & CAD (case) & Valve disease (case) & Pericarditis (case) & Cardiac tumor \\
\hline Lung cancer & 15 & $\mathrm{I}$ & $\mathrm{I}$ & $\mathrm{I}$ \\
Lung benign disease & 3 & & \\
Esophageal cancer & 2 & 2 & \\
Mediastinal disease & 3 & $\mathrm{I}$ & \\
Breast cancer & $\mathrm{I}$ (with VSD) & $\mathrm{I}$ & \\
Chest wall cavernous hemangioma & 10 & & \\
Gastrointestinal cancer & 4 & & \\
Biliary benign disease & 14 & 3 & $\mathrm{I}$ \\
Urinary cancer & $\mathrm{I}$ & 3 & \\
Gynecological disease & &
\end{tabular}

Abbreviations: CAD, coronary artery disease; VSD, ventricular septal defect. 
case; $\mathrm{CABG}$ and gastric antrum cancer resection in one case; $\mathrm{CABG}$ and distal radical gastrectomy (Belot type II) in one case; $\mathrm{CABG}$ and right half colon radical resection and ileum-colon anastomosis in three cases; CABG and colostomy in one case; $\mathrm{CABG}$ and transabdominal anterior resection of rectal cancer with Dixon type in two cases; $\mathrm{CABG}$ and liver cancer radical resection in one case; $\mathrm{CABG}$ and laparoscopic cholecystectomy in two cases; $\mathrm{CABG}$ and open cholecystectomy and appendectomy in one case; and CABG and cholecystectomy, common bile duct exploration, and $\mathrm{T}$ tube drainage in one case.

The heart and urinary system operations included CABG and right renal radical resection in seven cases (with inferior vena cava tumor thrombus removal in three cases); $\mathrm{CABG}$ and left renal radical resection in three cases; $\mathrm{CABG}$ and ventricular thrombus removal and ventricular aneurysm resection combined with right adrenal gland resection in one case; $\mathrm{CABG}$ and left renal and ureter radical resection in one case; emergency $\mathrm{CABG}$ and total cystectomy and ureterostomy in one case; and $\mathrm{CABG}$ and transurethral resection of bladder cancer in one case.

The heart and gynecological operations included mitral valve replacement, tricuspid annuloplasty, and radical hysterectomy in one case; aortic, mitral valve replacement, and hysterectomy in two cases; atrial tumor thrombus and inferior vena cava tumor thrombus removal and hysterectomy in one case; and CABG and double ovarian cyst resection and cervical polyp resection in one case.

\section{Operative incision}

The heart and thoracic operation incisions: 18 cases had median sternotomy for their cardiac and mediastinum or lung operations, two had median sternotomy combined with left thoracotomy, and four had median sternotomy with right thoracotomy. Two cases of CABG and lung cancer operations and one case of $\mathrm{CABG}$ and esophageal cancer operation were treated by left thoracotomy.

The heart and abdominal gastrointestinal operation incisions: one case had combined median sternotomy thoracoabdominal incision for his $\mathrm{CABG}$ and gastric cardia carcinoma resection, while the others had median sternotomy with abdominal rectus or paramedian incision or the laparoscopic approach.

The heart and urinary operation incisions: median sternotomy and left or right subcostal incisions were used for $\mathrm{CABG}$ and renal or adrenal cancer; median sternotomy and left abdominal oblique incision were used for $\mathrm{CABG}$ and the whole ureter resection; and median sternotomy and median incision of the lower abdomen were used for CABG and cystectomy.
The heart and gynecological operation incisions: median sternotomy and abdomen paramedian incision.

\section{Operative sequence}

Fifty-five cases were first treated by cardiac operation and nine cases by noncardiac operation.

\section{Cardiopulmonary bypass and intra-aortic balloon pump}

Cardiopulmonary bypass (CPB) was performed on 16 cases, of whom 13 were under cardiac arrest and three were under beating heart, including two cases of renal cancer resection with inferior vena cava tumor thrombus removal operation. The remaining one case of constrictive pericarditis with right lung cancer had pericardial stripping and right lower lobectomy ventricular fibrillation after the chest was closed and had to support circulation with CPB assist and postoperative intra-aortic balloon pump for 10 days.

\section{Operation mode of CABG}

Off-pump CABG was used for 49 cases, two of whom were followed by $\mathrm{CPB}$ to remove the inferior vena cava tumor thrombus. Four special cases who underwent CABG with $\mathrm{CPB}$, including one case of renal cancer with inferior vena cava tumor thrombus, one case of left lung cancer and left atrial tumor thrombus, one case of ventricular septal defect, and one case of left ventricular aneurysm and thrombus.

\section{Graft selection of CABG}

Bypass graft numbers were 1-4. Single left internal mammary artery (LIMA) was used to anastomose with left anterior descending in six cases; LIMA and radial artery were used for Y-shaped anastomoses in one case; the saphenous vein graft was used alone in 24 cases; and combined artery and vein grafts were used in 22 cases.

\section{Statistical method}

Kaplan-Meier method ${ }^{1}$ was used to make a statistical description of the survival time.

\section{Results Mortality}

Two patients died in hospital after the operations; thus, the hospital mortality rate was $3.1 \%$. The first patient with coronary heart disease, acute anterior wall myocardial infarction, and left ureteral cancer had a recurrence of bladder cancer 3 years after surgery. Although he accepted emergency simultaneous $\mathrm{CABG}$, bladder cystectomy, and ureterostomy, 
he died of acute cerebral infarction complicated with multiple organ failure on the 153rd day after operation. The other patient with chronic constrictive pericarditis and right lung cancer underwent pericardial stripping and right lung lower lobectomy. Ventricular fibrillation occurred after operation. CPB and then intra-aortic balloon pump were applied, resulting in acute renal failure. In spite of continuous hemofiltration treatment, he died on the tenth day postoperatively. The remaining 62 patients recovered and were discharged. Postoperative intensive care unit time was 1-153 days (mean 3 days). The postoperative hospital stay was 9-153 days (mean 15 days).

\section{Operation complications}

Total operation complications occurred in eleven cases, and the morbidity was $17.2 \%$. During the period of hospitalization, there was one case of postoperative hemorrhage without reexploration $(1.6 \%)$, two cases of pulmonary infection and hypoxemia $(3.1 \%)$, one case of hemorrhage of upper digestive tract $(1.6 \%)$, three cases of incisional infection (4.7\%), one case of the subphrenic abscess (1.6\%), and three cases of postoperative acute renal failure and hemofiltration (4.7\%).

\section{Follow-up}

Sixty-one cases were followed up from 1 month to 13 years and 10 months (the first patient was lost to follow-up). Eleven patients died within 10 months to 10 years during the follow-up, out of whom, nine died of tumor metastasis or recurrence, two died of other causes, but none died of cardiovascular events. Their time of death also varies: two cases died within 1 year to 2 years and 3 months after $C A B G$ and gastrointestinal cancer resection; five cases died within 10 months to 6 years and 4 months after heart and lung cancer operation; three cases died within 3 years and 8 months to 10 years after $\mathrm{CABG}$ and urinary cancer resection. The remaining 50 patients survived from 1 month to 13 years and 10 months during the follow-up. The mean survival time is $116.2 \pm 12.4$ months. The cumulative survival rate is $50.8 \%$ (Figure 1).

Eight patients survived in spite of metastasis or recurrence of cancer, including two cases after $\mathrm{CABG}$ and urinary operations, three cases after $\mathrm{CABG}$ and lung cancer operations, and three cases after $\mathrm{CABG}$ and gastrointestinal cancer resection. One patient who had mitral valve replacement and hysterectomy suffered heart failure and received medical treatment 1 month after the operation. One patient suffered unstable angina pectoris and was cured

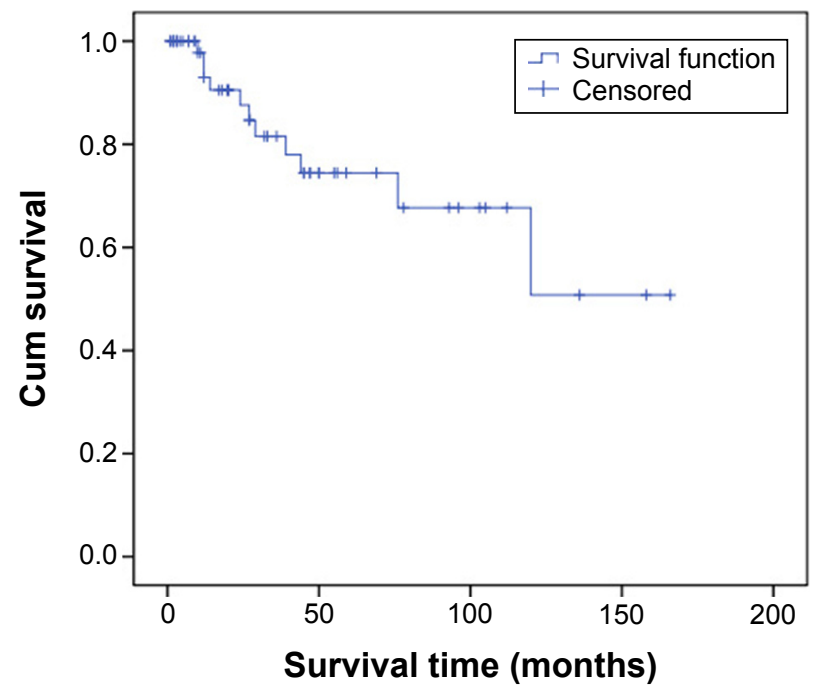

Figure I Simultaneous surgery patients' survival curve.

Notes: The mean survival time is II6.2 \pm 12.4 months. The cum survival rate is $50.8 \%$. Abbreviation: cum, cumulative.

by percutaneous coronary intervention (PCI) 3 years after $\mathrm{CABG}$ and lung cancer surgery. The remaining patients did not encounter cardiovascular events (acute myocardial infarction, heart failure, sustained ventricular tachycardia, ventricular fibrillation, sudden cardiac death, etc). One patient who had mechanical prosthetic valve replacement showed excessive anticoagulation, leading to cerebral hemorrhage 3 months after the operation. One patient who underwent thoracoabdominal incision was diagnosed with subxiphoid incisional hernia 1 year later and 'was operated on successfully.

\section{Discussion}

It is really a difficult issue to handle patients who need cardiac and noncardiac surgical operations at the same time, especially those early malignant tumor patients with severe coronary artery diseases, as contraindications to each other previously. $^{2-4}$ The question of how best to deal with these patients still remains open. Either a one-stage or a two-stage operation are possibilities; there are several arguments for and against both treatment strategies. A two-stage procedure means two anesthetic agents for the patient, twice the surgical trauma, a longer overall stay in hospital, and delay in the tumor resection in the tumor patients. Additionally, it doubles the preoperative stress and postoperative pain and leads to higher costs of treatment. On the other hand, simultaneous surgery may be an excessive trauma, for the anesthesia and operation time will be prolonged and the risk of postoperative bleeding will increase related to heparinization. 
CAD and noncardiac diseases are common occurrences found in in these cases mentioned above. If PCI is performed first, the intensive antiplatelet therapy and this usually needs for 3-6 months. Some patients need intensive anticoagulation for 12 months. Patients with coronary stents who have undergone surgery are at high risk for hemorrhagic complications. A prospective research showed that the postoperative hemorrhagic complication was $9.5 \%$ during the first 30 days following the surgery. Mortality rate in patients with a hemorrhagic complication was $12 \%$. Independent postoperative factors associated with hemorrhagic complications are identified as a high and intermediate bleeding risk procedure and the use and dose of anticoagulants. ${ }^{5}$ Adverse outcomes of noncardiac surgery soon after coronary stenting are associated with a major risk of operative myocardial ischemia, even in case the recommended 6 week interval. ${ }^{6,7}$ A 3-month delay between PCI and surgical intervention has been recommended to minimize the risk of in-stent thrombosis. ${ }^{8}$ Furthermore, another study supports a significant decrease in major adverse cardiac events when surgery is performed 1 year after drug-eluting stents placement. ${ }^{9}$ In these cancer patients, surgery should not be postponed for months. Marcucci et $\mathrm{al}^{7}$ recommended a preoperative revascularization limited to balloon angioplasty without stenting. Once the patient has recovered from the noncardiac operation, a definitive PCI could be performed.

Another choice, if CABG is done first, is that the postoperative recovery period is prolonged. During this period of time, if the malignant tumor continues growing, the best chance of operation may be missed. If tumor resection is conducted first, the perioperative risk of myocardial ischemia or infarction and cardiac dysfunction may increase, and so does the perioperative mortality. For the heart valve disease patients, if they need noncardiac surgery, the valve diseases should be handled before elective surgical procedures according to the severity of the symptoms and stenosis/ regurgitation, which needs valve interventions (replacement or repair).${ }^{10}$ With the gradual improvement of surgical techniques, simultaneous cardiac and noncardiac operations become possible. In fact, simultaneous surgery has already been proposed in the 1980s for combined treatment of pulmonary neoplasia and cardiac surgical disorders. Several years later, Danton et al ${ }^{11}$ rejected the initial concerns about oncological outcomes after simultaneous surgery for lung cancer, stating that simultaneous pulmonary and cardiac surgery was associated with acceptable postoperative morbidity and mortality rates with similar long-term survival results. Up to now, simultaneous surgeries of heart diseases and cancer have been reported worldwide. Simultaneous cardiac surgery and pulmonary resection for malignancy have become almost a standard treatment of patients who require heart and lung surgery. ${ }^{12-20}$ In recent years, cardiac operation has not been limited to CABG. It is also seen in the heart valve replacement and aortic aneurysm operation. ${ }^{14,19,20} \mathrm{~A}$ large number of clinical cases wordwide with confirmed curative effect, prognosis, and long-term follow-up are desirable. However, such large domestic reports are rare in the People's Republic of China. ${ }^{21-24}$

According to our research, the simultaneous operation indications are 1) preoperative estimation of malignant tumors in early- and middle-stage patients, who are able to tolerate radical resection, without distant metastasis, and who suffer from CAD and a complication of myocardial infarction with $\mathrm{CABG}$ indications, at the same time; 2) single- or double-vessel disease in CAD and tumor patients who are able to take PCI but need tumor resection without delay; 3) patients whose tumors require resection, whose heart valve diseases need replacement or repair at the same time, and whose heart conditions can tolerate simultaneous surgeries (with improved heart function grades II-III by medical therapy); 4) CAD patients with late-stage renal cancer and inferior vena cava tumor thrombus can have prolonged life after tumor resection, and long-term prognosis is relatively acceptable, ${ }^{25,26} 5$ ) neoplasm is found in cardiac operation; 6) patients are expected to tolerate simultaneous cardiac and noncardiac benign disease surgeries.

Cardiac surgery often uses median sternotomy, with the incision fully exposed to the heart. Median sternotomy can be used in a variety of heart surgeries and facilitate the timely establishment of CPB as well, when needed. Median sternotomy is also suitable for certain simultaneous thoracic surgeries. In the combined abdominal operation, different abdominal incisions are selected according to the location and extent of operation. Technical problems may be encountered regarding a lung malignant lesion through a median sternotomy. It has been reported that sternotomy, the preferred approach in cardiac operation, is also suitable for right lung resections and occasionally left upper lobectomy. In cases of right pneumonectomy, sternotomy even facilitates the formation of a bronchial stump. Exposure of upper mediastinal lymph nodes, especially subcarinal nodes, is good but requires a transpericardial approach. The dissection of paraesophageal lymph nodes on both sides after sternotomy is more demanding than from standard thoracotomy access. ${ }^{11,15,16,24}$ Left lower lobectomy is the most difficult lung resection to perform through a median sternotomy. Retraction 
of heart during this procedure may lead to hemodynamic compromise and arrhythmias. Opening the right pleura and pericardium widely and rotating the heart in an anticlockwise fashion can improve the exposure. Occasionally, the left anterolateral thoracotomy incision is applied for the CABG and left lung or esophagus operation. But left thoracotomy limits CABG operation on a beating heart to the left coronary artery and its branches. Alternatively, surgeons can have a separate thoracotomy incision after cardiac surgery though median sternotomy. ${ }^{16,24}$ Finally, the size and localization of the tumor determine the planning of a combined cardiac and thoracic incision.

In general, cardiac operation should be done first. With myocardial blood supply improved or valve handled, intraoperative myocardial ischemia and cardiac dysfunction can be effectively avoided, thus increasing patients' tolerance of the following operation. Secondly, cardiac surgery is an aseptic surgery. The others are relative contaminated surgeries. On the other hand, thymoma or thymic cysts in front of the heart have to be removed first so as to avoid barriers in cardiac operation and reduce the wound bleeding after heparinization.

$\mathrm{CPB}$ is an important device in cardiac surgery. Yet, multiple disadvantages of $\mathrm{CPB}$ have been reported as follows: 1) increased risk of bleeding due to heparinization; 2) activation of the systemic inflammatory response syndrome (SIRS), impairing the immune response against the growth of tumor cells and infections; 3 ) the risk of edema in the residual lung tissue; and 4) the possibility of the dissemination of neoplastic cells. ${ }^{18}$ At present, the effects of CPB on tumor growth are still a matter of debate. There is enough evidence to suggest that $\mathrm{CPB}$ exhibits an impact on many aspects of immunity; however, there is neither a significant increase in morbidity and mortality of cardiac surgery with CPB nor an effect on the tumor growth or the spread of malignancy in cancer patients, even in an active stage. It seems that the survival of cancer patients who undergo cardiac surgery is more closely related with the progression of the tumor than the surgical procedure. ${ }^{27}$

Although CPB is essential in open heart surgeries for the valve replacement and atrial myxoma removal, for $\mathrm{CABG}$, we recommend the off-pump technique. This technique can reduce intraoperative heparin dose, reduce bleeding and postoperative errhysis during tumor resection, and, at the same time, reduce the effect of inflammatory mediators produced by $\mathrm{CPB}$. CPB is often used in the therapy of special diseases. For instance, the surgery of the left ventricular aneurysm and thrombus in one $\mathrm{CAD}$ patient was conducted under $\mathrm{CPB}$; the inferior vena cava tumor thrombus in three renal cancer patients was removed with the aid of $\mathrm{CPB}$, including using $\mathrm{CPB}$ with deep hypothermia and low flow in one CAD patient and doing $\mathrm{CABG}$ anastomosis during rewarming. One $\mathrm{CABG}$, left pneumonectomy, and left atrial tumor thrombus removal case used CPB by aortic and pulmonary artery cannula.

The CABG graft strategies are selected according to the coronary artery conditions, natural history of benign and malignant noncardiac diseases, malignant degree and stage, the expected survival rate, operative incision factors, etc. The mixed application of arteries and veins is used in most multivessel diseases; LIMA is most commonly used in single anterior descending artery disease; left anterior lateral thoracotomy can also harvest LIMA. Saphenous vein graft can be used alone as grafts in patients with high-degree malignant or late-stage diseases or in patients who are expected to have chest radiotherapy. Although its 10 -year patency rate is only $40 \%-60 \%,{ }^{28}$ it can ensure the intra-operative and postoperative coronary blood supply, which is of importance for the recovery and survival of the tumor patients, as it can help them to avoid occurrences of cardiovascular events within their life expectancy. Meanwhile, it can save time (compared with the harvesting of LIMA), reduce the wound surface bleeding, and ensure the integrity of the pleura, which is beneficial to the recovery of the respiratory function after operation.

In our study the hospital mortality was $3.1 \%$. In consideration of the type and severity of diseases (such as acute myocardial infarction, myocardial atrophy, heart and multiple organ failures, simultaneous excessive trauma, etc), it is possible and acceptable to perform simultaneous surgeries on patients with both cardiac and noncardiac diseases when considering the hospital mortality of $0 \%-6.5 \%$ as reported by other researchers. ${ }^{12,18}$ There is no patient required reexploration for bleeding. The wound complication was comparatively low. Respiratory complications, such as pulmonary infection and hypoxemia, can be also seen, because postoperative acute renal failure and hemofiltration mainly depend on the preoperative renal function and the degree of damage during the simultaneous surgery. What matters is that surgeons must take into account every single operative risk related to every single problem. They must decide in the first place whether there is a necessity for simultaneous surgery or whether there is contraindication. In order to select the best surgical strategy for each patient, a complete preoperative examination and an accurate estimation of combined risks should be carried out before treatment planning.

At present, the survival rate is still determined by the tumor stage. ${ }^{29,30}$ The long-term follow-up found that in 
this group of patients, fewer postoperative cardiac events occurred, and metastatic spread of cancer still had a greater impact on prognosis. The poor prognosis of patients suffered lung cancer or gastrointestinal cancer and the relative good prognosis of patients suffered renal cancer even with inferior vena cava tumor thrombus suggest that the prognosis mainly depends on the biological behavior of the different malignant tumors. As some researchers have demonstrated a 5 -year survival rate $<40 \%$ in patients who had heart and lung operations, our result is a quite favorable experience. ${ }^{13}$

\section{Conclusion}

Simultaneous surgery is feasible and safe for patients suffering from cardiac and noncardiac diseases, especially for those early malignant tumor patients with severe CAD. The short-term curative effect is quite satisfactory, and both mortality and morbidity are low. The long-term survival of the patients mainly depends on the type and stage of the tumor. In this study, due to the small number of cases, the research was retrospective. We did not use any control group for comparison; thus, we were obliged to refer to the historical data from published reports. This study will continue to collect data and summarize the regularity and efficacy of the simultaneous surgery.

\section{Acknowledgment}

The research is done in honor of the 100th anniversary of Peking University First Hospital.

\section{Disclosure}

The authors report no conflicts of interest in this work.

\section{References}

1. Jager KJ, van Dijk PC, Zoccali C, Dekker FW. The analysis of survival data: the Kaplan-Meier method. Kidney Int. 2008;74(5):560-565.

2. Azpitarte J. Editorial: cardiac surgery in the cancer patient. Rev Esp Cardiol. 2008;61(4):349-351.

3. Mann DL, Krone RJ. Editorial: cardiac disease in cancer patients: an overview. Prog Cardiovasc Dis. 2010;53(2):80-87.

4. Chan J, Rosenfeldt F, Chaudhuri K, Marasco S. Cardiac surgery in patients with a history of malignancy: increased complication rate but similar mortality. Heart Lung Circ. 2012;21(5):255-259.

5. Albaladejo P, Charbonneaua H, Samamac CM, et al. Bleeding complications in patients with coronary stents during non-cardiac surgery. Thromb Res. 2014;134(2):268-272.

6. Kaluza GL, Joseph J, Lee JR, Raizner ME, Raizner AE. Catastrophic outcomes of noncardiac surgery soon after coronary stenting. J Am Coll Cardiol. 2000;35(5):1288-1294.

7. Marcucci C, Chassot PG, Gardaz JP, et al. Fatal myocardial infarction after lung resection in a patient with prophylactic preoperative coronary stenting. Br J Anaesth. 2004;92(5):743-747.

8. Chassot PG, Delabays A, Spahn DR. Preoperative evaluation of patients with, or at risk of, coronary artery disease undergoing non-cardiac surgery. Br J Anaesth. 2002;89(5):747-759.

9. Hollis RH, Graham LA, Richman JS, Deierhoi RJ. Adverse cardiac events in patients with coronary stents undergoing noncardiac surgery: a systematic review. Am J Surg. 2012;204(4):494-501.
10. Fleisher LA, Fleischmann KE, Auerbach AD, et al; American College of Cardiology; American Heart Association. 2014 ACC/AHA Guideline on perioperative cardiovascular evaluation and management of patients undergoing noncardiac surgery: a report of the American College of Cardiology/American Heart Association task force on practice guidelines. J Am Coll Cardiol. 2014;64(22):e77-e137.

11. Danton MH, Anikinb VA, McManusb KG, McGuiganb JA, Campalania G. Simultaneous cardiac surgery with pulmonary resection: presentation of series and review of literature. Euro J Cardiothorac Surg. 1998;13(6): $667-672$.

12. Riviere AB, Knaepen P, Swieten HV, Vanderschueren R, Ernst J, Bosch JV. Concomitant open heart surgery and pulmonary resection for lung cancer. Euro J Cardiothorac Surg. 1995;9(6):310-314.

13. Rao V, Todd TR, Weisel RD, et al. Results of combined pulmonary resection and cardiac operation. Ann Thorac Surg. 1996;62(2):342-347.

14. Davydov MI, Akchurin RS, Gerasimov SS, et al. Simultaneous operations in thoraco-abdominal clinical oncology. Euro $J$ Cardiothorac Surg. 2001;20(5):1020-1024.

15. Mariani MA, Boven WJ, Duurkens VA, Ernst SM, Swieten HA. Combined off-pump coronary surgery and right lung resections through midline sternotomy. Ann Thorac Surg. 2001;71(4):1343-1344.

16. Saxena $P$, Tam RK. Combined off-pump coronary artery bypass surgery and pulmonary resection. Ann Thorac Surg. 2004;78(2):498-501.

17. Dyszkiewicz W, Jemielity MM, Piwkowski CT, Perek B, Kasprzyk M. Simultaneous lung resection for cancer and myocardial revascularization without cardiopulmonary bypass (off-pump coronary artery bypass grafting). Ann Thorac Surg. 2004;77(3):1023-1027.

18. Schoenmakers MC, Boven WJ, Bosch J, Swieten HA. Comparison of on-pump or off-pump coronary artery revascularization with lung resection. Ann Thorac Surg. 2007;84(2):504-509.

19. Sabol F, Toporcer T, Kolesár A, Belák J, Luczy J. Case report: surgical management of a patient with combined heart pathologies and lung cancer. A simultaneous coronary artery bypass surgery, aortic valve replacement, tricuspid valve repair and pulmonary resection. Cor Vasa. 2014;56: e75-e79.

20. Santavy P, Szkorupa M, Bohanes T, Lonsky V. Simultaneous cardiac surgery with pulmonary resection. Cor Vasa. 2015;57(2):e82-e85.

21. Wang J, Xiao F, Tao L. [One-stage coronary artery bypass grafting and tumor resection.] Chin J Surg. 2002;40:394. Chinese.

22. Yang Y, Xiao F, Wang J, Li J, Zhou LQ. [Simultaneous surgery in patients suffering from tumor combined with coronary artery disease.] J Pek Univ Health Sci. 2007;39:416-419. Chinese.

23. Song B, Xiao F, Li Y. [Simultaneous tumor resection and cardiac operation.] Chin J Clin Oncol Rehabil. 2007;14:509-511. Chinese.

24. Zhang YY, Wu YH, Yuan B, et al. Case Report: coronary artery bypass grafting with concomitant resection for carcinoma of lung. J Biomed Res. 2010;24(1):77-80.

25. Franke UFW, Wahlersa T, Wittwera T, Schubert J. Case report: renal carcinoma with caval vein infiltration and triple coronary disease: onestage surgical management. Euro J Cardiothorac Surg. 2001;20(4): 877-879.

26. Wang J, Xiao F, Tao L, et al. [Cardiovascular surgical technique in the treatment of urological tumor with thrombosis involving the inferior vena cava.] J Pek Univ Health Sci. 2001;33:23-25. Chinese.

27. Carrascal Y, Gualis J, Arevalo A, et al. Cardiac surgery with extracorporeal circulation in cancer patients: influence on surgical morbidity and mortality, and on survival. Revist Espan Cardio. 2008;61(4):369-375. English Edition.

28. Sergeant P, Blackstone E, Meyns B. Validation and interdependence with patient-variables of the influence of procedural variables on early and late survival after CABG. Leuven Coronary Surgery Porgram. Euro J Cardiothorac Surg. 1997;12(1):1-19.

29. Mistiaen WP, Cauwelaert PV, Muylaert P, Wuyts F, Harrisson F, Bortier H. Effect of prior malignancy on survival after cardiac surgery. Ann Thorac Surg. 2004;77(5):1593-1597.

30. Rausei S, Chiappa C, Franchin M, et al. Malignancy as a risk factor in single-stage combined approach for simultaneous elective surgical diseases. Int J Surg. 2013;11(suppl 1):S84-S89. 


\section{Publish your work in this journal}

Patient Preference and Adherence is an international, peer-reviewed, open access journal that focuses on the growing importance of patient preference and adherence throughout the therapeutic continuum. Patient satisfaction, acceptability, quality of life, compliance, persistence and their role in developing new therapeutic modalities and compounds to optimize

clinical outcomes for existing disease states are major areas of interest for the journal. This journal has been accepted for indexing on PubMed Central. The manuscript management system is completely online and includes a very quick and fair peer-review system, which is all easy to use. Visit http://www. dovepress.com/testimonials.php to read real quotes from published authors.

Submit your manuscript here: http://www.dovepress.com/patient-preference-and-adherence-journal 